and let $z=e M N(r+1)^{-\frac{1}{2}}$ this becomes

$$
\left|\sum_{r+1}^{N} a_{n}\right|<\frac{1}{[2 \pi(r+1)]^{1 / 2}} \frac{z^{r+1}}{1-z e^{-1 / 2}} .
$$

Therefore if $M$ is sufficiently small, that is, if $z \ll 1$ for some $r<N$, it becomes feasible to approximate (8) by

$$
B^{-1} \approx I+\frac{\sum_{0}^{r} A_{n}}{\sum_{0}^{r} a_{n}} .
$$

If we do so we are assured that the error in the denominator will be no larger than (16) and similarly the errors in the elements of the numerator will be no greater than

$$
\frac{(r+1)^{1 / 2}}{N(2 \pi)^{1 / 2}} \cdot \frac{z^{r+1}}{1-z[(r+1) / e r]^{1 / 2}} .
$$

\title{
REFERENCES
}

1. J. S. Frame, $A$ simple recursion formula for inverting a matrix, Abs. 471, Bull. AMS 65, 1045 (1949). See also, Henry E. Fettis, Quart. Appl. Math. 8, 207 (1950).

2. Paul S. Dwyer, Linear computations, Wiley, 1951, pp. 223. See also, Chrystal, Textbook of algebra, Chelsea, 1952, Vol. I, pp. 437.

3. Lovitt, Linear integral equations, Dover, 1950, Chap. III.

\section{THE ELASTIC SPHERE UNDER CONCENTRATED TORQUES ${ }^{1}$}

\section{BY ALFRED HUBER (University of Maryland)}

1. Introduction. In this note we consider an elastic sphere subject to two concentrated torques acting at the end points of a diameter. The stress function occurring in this problem may be considered as a generalized axially symmetric potential ${ }^{2}$. This viewpoint enables us to obtain the solution by a simple potential-theoretic argument.

The notation used in this article is the same as that used by Timoshenko in [6].

2. Stress function. We introduce cylindrical coordinates $(r, \theta, z)$ and consider the elastic sphere $z^{2}+r^{2} \leqq 1$, subject to unit torques acting at the points $r=0, z= \pm 1$. All relevant quantities appear in a meridian $(z, r)$-plane. According to the theory of torsion of circular shafts we must find a stress function $\Phi(z, r)$ satisfying the following conditions: $\Phi$ is to be a solution of

$$
\frac{\partial^{2} \Phi}{\partial z^{2}}+\frac{\partial^{2} \Phi}{\partial r^{2}}-\frac{3}{r} \frac{\partial \Phi}{\partial r}=0
$$

defined and bounded in the half-circular region $H=\left[r^{2}+z^{2}<1\right] \cap[r>0]$, assuming the boundary value 0 on the open diameter, $1 / 2 \pi$ on the open circular boundary. This problem shall in the sequel be referred to as problem $(\mathrm{P})$.

${ }^{1}$ Received May 1, 1954. This research was supported by the United States Air Force through the Office of Scientific Research of the Air Research and Development Command.

${ }^{2}$ For the definition of this concept the reader is referred to [8]. 
We consider $u(z, r)=2 \pi \Phi(z, r)-1$. This function vanishes on the half-circle and can be continued analytically beyond it by the relation

$$
u(z, r)=-\rho^{3} u\left(z \rho^{-2}, r \rho^{-2}\right),
$$

where $\rho=\left(z^{2}+r^{2}\right)^{1 / 2}$. For, by means of the correspondence principle (A. Weinstein [7]) the function $v(z, r)=r^{-4} u(z, r)$ is an axially symmetric potential in seven-dimensional space, considered in a meridian plane. The continuation (2.2) follows immediately from the classical reflection principle for harmonic functions, applied to $v$.

$u$ is now defined in the whole half-plane $r>0$, satisfies (2.1), assumes known boundary values on $r=0$ and $u=O\left(\rho^{3}\right)$ at infinity. Such a function is obtained as a generalized Poisson integral (A. Weinstein [8])

$$
u=\frac{3}{4} r^{4}\left\{\int_{-\infty}^{-1}(-\zeta)^{3} d^{-5} d \zeta+\int_{-1}^{+1}(-1) d^{-5} d \zeta+\int_{+1}^{+\infty} \zeta^{3} d^{-5} d \zeta\right\}
$$

where $d=\left[(z-\zeta)^{2}+r^{2}\right]^{1 / 2}$. An elementary computation yields

$$
\begin{aligned}
\Phi=\frac{1}{8 \pi}\left[4-\left(1-\rho^{2}\right)\left(a^{-3}+b^{-3}\right)\left(2 \rho^{4}+8 z^{2}+5 r^{2}\right.\right. & +2) \\
& \left.-6 z\left(a^{-3}-b^{-3}\right)\left(1-\rho^{4}\right)\right],
\end{aligned}
$$

where $a=\left[(z+1)^{2}+r^{2}\right]^{1 / 2}, b=\left[(z-1)^{2}+r^{2}\right]^{1 / 2}$ and $\rho=\left(z^{2}+r^{2}\right)^{1 / 2}$. $\Phi$ has the required properties. This is implied by the construction and can also be directly verified.

We mention, that (2.3) could also be inferred from a formula of M. Olevskii [4], which was derived in a different way. Olevskii solved a more general problem whose solution is also accessible to the above potential-theoretic approach. Related results have recently been announced by $\mathrm{P}$. Brousse $[1,2]$.

3. Stresses and displacements. The stresses are obtained by differentiation:

$$
\begin{aligned}
& \begin{aligned}
\tau_{\theta z}=\frac{1}{r^{2}} \frac{\partial \Phi}{\partial r}=\frac{3}{4 \pi r} & \left(a^{-3}+b^{-3}\right)\left(2 \rho^{4}+2 \rho^{2}+z^{2}-1\right) \\
+ & \frac{3 z \rho^{2}}{\pi r}\left(a^{-3}-b^{-3}\right)+\frac{3}{8 \pi r}\left(a^{-5}+b^{-5}\right)\left(1-\rho^{2}\right)\left(2 \rho^{4}+8 z^{2}+5 r^{2}+2\right) \\
& +\frac{9 z}{4 \pi r}\left(1-\rho^{4}\right)\left(a^{-5}-b^{-5}\right),
\end{aligned} \\
& \begin{aligned}
& \tau_{r \theta}=-\frac{1}{r^{2}} \frac{\partial \Phi}{\partial z}=-\frac{3 z}{4 \pi r^{2}}\left[\left(a^{-3}+b^{-3}\right)\left(\rho^{4}+2 \rho^{2}+z^{2}\right)\right. \\
& \quad\left.\quad\left(a^{-3}-b^{-3}\right)\left(3 z \rho^{2}+z\right)\right]-\frac{3}{8 \pi}\left(1-\rho^{2}\right)\left[(1+z) a^{-5}-(1-z) b^{-5}\right] .
\end{aligned}
\end{aligned}
$$

Both components vanish on the axis of symmetry. In the equator plane $(z=0)$ the above expressions reduce to

$$
\tau_{\theta z}=\frac{3 r}{4 \pi}\left(2 r^{4}+5 r^{2}+5\right)\left(1+r^{2}\right)^{-5 / 2}, \quad \tau_{r \theta}=0 .
$$


Figure 1 shows the stress distribution in the equator plane, the dotted line indicating the respective stresses for the circumscribed cylinder. Figure 2 respresents the stresses in the cross-section $z=-0.5$.
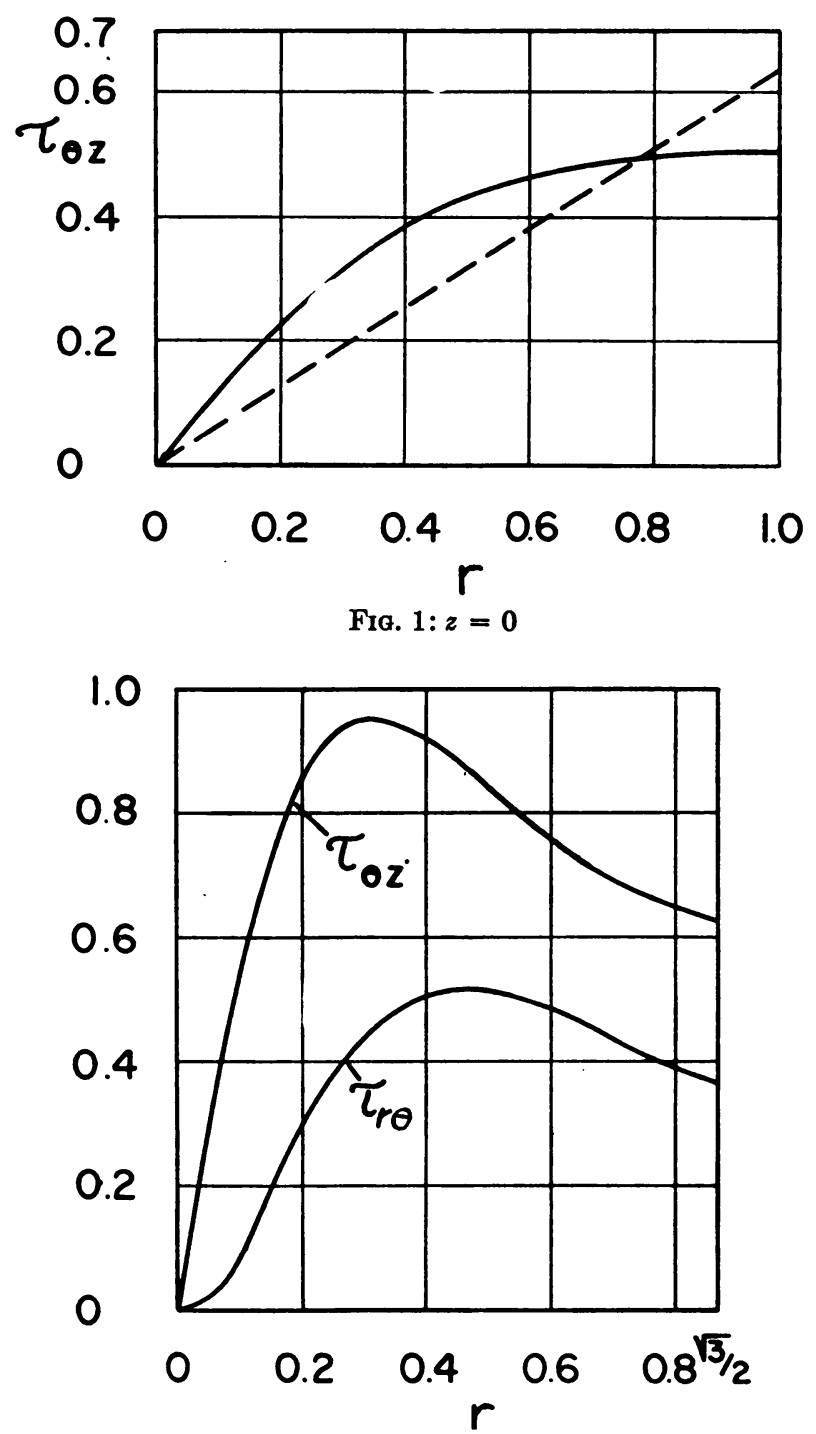

Fig. 2: $z=-0.5$

In the general case of the sphere $z^{2}+r^{2} \leqq R^{2}$, subject to torques of magnitude $M$, the stresses at $(z, r)$ can be computed by multiplying with $M R^{-3}$ the respective quantities for $M=R=1$, taken at the point $\left(z R^{-1}, r R^{-1}\right)$.

In order to determine the displacements for $M=R=1$, we observe that $u=w=0$ and $v=r \Psi(z, r)$ (see e.g. [6]), where $\Psi$ satisfies the equation

$$
\frac{\partial^{2} \Psi}{\partial z^{2}}+\frac{\partial^{2} \Psi}{\partial r^{2}}+\frac{3}{r} \frac{\partial \Psi}{\partial r}=0
$$


and is connected with $\Phi$ by the generalized Cauchy-Riemann equations

$$
G r^{3} \frac{\partial \Psi}{\partial z}=\frac{\partial \Phi}{\partial r}, \quad G r^{3} \frac{\partial \Psi}{\partial r}=-\frac{\partial \Phi}{\partial z},
$$

$G$ denoting the modulus of shear. $\Psi$ is determined up to an additive constant by these relations. Since $\Psi=$ const. on $z=0$ because of $\partial \Psi / \partial r=\partial \Phi / \partial z=0$, we may define

$$
\Psi(0, r) \equiv 0 .
$$

It follows from (3.2) and (3.3) that

$$
\Psi(z, r)=G r^{-3} \int_{0}^{z} \frac{\partial \Phi}{\partial r} d z
$$

It can easily be seen, without carrying out explicitly the cumbersome integration, that $\Psi(z, r)$ can also be represented as a closed expression of elementary functions.

4. Uniqueness of the solution. Let $\Phi_{1}$ and $\Phi_{2}$ be two solutions of problem (P). The function $f=\Phi_{1}-\Phi_{2}$ satisfies (2.1), is bounded in $H$ and assumes the value 0 at all points of the boundary with the possible exception of $A(-1,0)$ and $B(+1,0)$.

We first show that $f$ approaches 0 also at $A$ and $B$. Let $f^{*}(z, r)$ denote a solution of (2.1), defined in the quarter-circle $Q=H \cap[z>0]$, with boundary values $f$ on $z=0$ $(0 \leqq r \leqq 1), 0$ elsewhere. $f^{*}$ exists; an explicit representation for $f^{*}$ in terms of the boundary values could easily be derived using the methods of Sec. 2 . We now consider the function $g=f-f^{*}$ which satisfies (2.1), is bounded in $Q$ and vanishes on the boundary except possibly at $B$. By an inversion with respect to the circle of radius 1 and center $B, Q$ is mapped on $Q_{1}$, an infinite subdomain of the half-plane $r>0$. The function $g_{1}$, defined in $Q_{1}$ by the relation

$$
g_{1}(z-1, r)=b^{3} g\left((z-1) b^{-2}, r b^{-2}\right),
$$

where $b=\left[(z-1)^{2}+r^{2}\right]^{1 / 2}$, satisfies $(2.1)$ in $Q_{1}$ and vanishes on the finite boundary. Furthermore, $g_{1}=O\left(\rho^{3}\right)$ for $\rho \rightarrow \infty$. Applying the Phragmén-Lindelöf theorem in [3] to both $g_{1}$ and $-g_{1}$, we conclude that $g_{1} \equiv 0$ throughout $Q_{1}$. Hence $g \equiv 0$ in $Q$. It follows that $f$ approaches 0 at $B$. The same is true for $A$.

Since $f$ is continuous and identically 0 on the whole boundary of $H$ we infer from the maximum-minimum principle that $f \equiv 0$ in $H$. This proves the uniqueness.

5. Discussion of singularities. Next we demonstrate that the solution of problem (P) yields the right type of singularity for the stresses at $A$ and $B$. The state of stress corresponding to two concentrated unit torques in these points can be defined by the following limiting process. We subject the sphere to torsion by two homogeneous distributions of shearing stresses, of resulting moment 1 each, acting on the spherical caps. $S_{A}=\left[z^{2}+r^{2}=1\right] \cap[|z+1|<1 / n]$ and $S_{B}=\left[z^{2}+r^{2}=1\right] \cap[|z-1|<1 / n]$, $n=1,2,3, \cdots$. Let $\Phi_{n}$ be the corresponding stress function, $\tau_{\theta z}^{n}$ and $\tau_{r \theta}^{n}$ the stresses. Their limits, as $n \rightarrow \infty$, define the state of stress due to concentrated unit torques in $A$ and $B$.

Because of the maximum principle we have $\Phi_{n} \leqq 1 / 2 \pi$, the boundary values being continuous and satisfying the same inequality. By a similar argument we see that $\left\{\Phi_{n}(z, r)\right\}$ is an increasing sequence. Therefore, it converges to a limit function $\Phi(z, r)$. The convergence is uniform in every compact subdomain of $H$ and the derivatives of $\Phi_{n}$ tend to the derivatives of $\Phi$. It can be verified without difficulty, that $\Phi$ is the stress 
function of the limit stresses and a solution of problem (P). Because of the uniqueness $\Phi$ is given by (2.4).

This verification as well as the uniqueness proof are by no means superfluous. In fact, the solution ceases to be unique if the condition of boundedness is dropped. Consider for example the function

$$
\Phi^{*}(z, r)=r^{4}\left(1-z^{2}-r^{2}\right)\left[(z-1)^{2}+r^{2}\right]^{-7 / 2},
$$

which satisfies (2.1) in $H$ and assumes the boundary value 0 everywhere, except at $B$, where $\Phi^{*}=O\left(b^{-2}\right)$. This stress function corresponds to a singular self-equilibrated system of forces at $B$. Superposing (2.4) and (4.2) we obtain a function which fulfills all conditions of problem (P) except boundedness. In the terminology of E. Sternberg and F. Rosenthal [5], who treated the case of concentrated loads, it is a "pseudosolution" of the physical problem.

\section{References}

[1] P. Brousse, Sur un probleme de Dirichlet singulier, Compt. rend. Acad. Sci. Paris 236, 1731-1732 (1953).

[2] P. Brousse, Quelques propriêtés des intégrales d'une classe d'équations singulières dans certains domaines, Compt. rend. Acad. Sci. Paris 237, 1381-1383 (1953).

[3] A. Huber, A theorem of Phragmen-Lindelöf type, Proc. Am. Math. Soc. 4, 852-857 (1953).

[4] M. Olevskii, Solution of the Dirichlet problem for the equation $\Delta u+\left(p / x_{n}\right) \partial u / \partial x_{n}=\rho$ for a hemispherical region, Doklady Akad. Nauk SSSR (N. S) 64, 767-770 (1949).

[5] E. Sternberg and F. Rosenthal, The elastic sphere under concentrated loads, J. Appl. Mechanics 19, 413-421 (1952).

[6] S. Timoshenko, Theory of elasticity, New York, 1934, pp. 276-284.

[7] A. Weinstein, Discontinuous integrals and generalized potential theory, Trans. Am. Math. Soc. 63, 342-354 (1948).

8] A. Weinstein, Generalized axially symmetric potential theory, Bull. Am. Math. Soc. 69, 20-38 (1953).

\section{ON SUBHARMONIC SYNCHRONIZATION OF NEARLY-LINEAR SYSTEMS*}

\section{BY HIRSH COHEN (Carnegie Institute of Technology)}

When a linear system is driven by an external force, one has become accustomed to expect a frequency response related to the combination of the driving frequency and the natural frequency of the system. If one of the elements of the system is non-linear, but only slightly so, we may expect the frequency of the periodic response to behave like that of a linear system. For a physical system which is described by the non-linear differential equation

$$
\ddot{y}+\epsilon f(y) \dot{y}+\omega_{0}^{2} y=F(t),
$$

where $y$ is of the order unity, and $\epsilon$ is very much less than unity, this is true for all but a small interval in the range of driving frequencies. It has been shown in several special cases, notably for the van der Pol equation [1], that if this system is driven by a force $A \cos (\omega t+\varphi)$, the periodic response of the system for values of $\omega^{2}-\omega_{0}^{2}$ small, say of order $\epsilon$, is not a combination or beat frequency, but rather a function with frequency

*Received May 10, 1954. 